\title{
Real-Time Unsteady Loads Measurements Using Hot-Film Sensors
}

\author{
Arun S. Mangalam* \\ Tao of Systems Integration, Inc., Pomona, CA, 91768, USA \\ and \\ Timothy R. Moes ${ }^{\dagger}$ \\ NASA Dryden Flight Research Center, Edwards, CA, 93523, USA
}

\begin{abstract}
Several flight-critical aerodynamic problems such as buffet, flutter, stall, and wing rock are strongly affected or caused by abrupt changes in unsteady aerodynamic loads and moments. Advanced sensing and flow diagnostic techniques have made possible simultaneous identification and tracking, in real-time, of the critical surface, viscosity-related aerodynamic phenomena under both steady and unsteady flight conditions. The wind tunnel study reported here correlates surface hot-film measurements of leading edge stagnation point and separation point, with unsteady aerodynamic loads on a NACA 0015 airfoil. Lift predicted from the correlation model matches lift obtained from pressure sensors for an airfoil undergoing harmonic pitchup and pitchdown motions. An analytical model was developed that demonstrates expected stall trends for pitchup and pitchdown motions. This report demonstrates an ability to obtain unsteady aerodynamic loads in real-time, which could lead to advances in air vehicle safety, performance, ride-quality, control, and health management.
\end{abstract}

\section{Nomenclature}

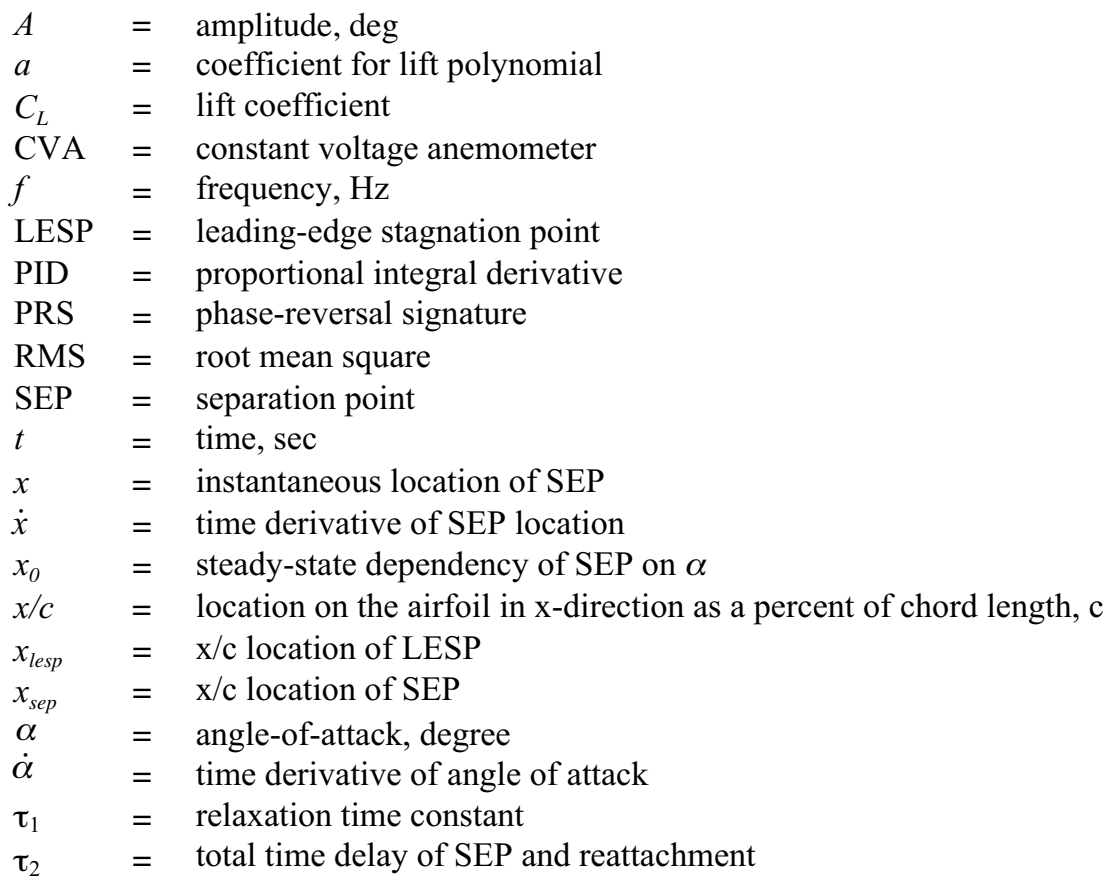

\footnotetext{
* Aerospace Engineer, 3660 West Temple Avenue, Suite 1340, Pomona, CA 91768, Member.

${ }^{\dagger}$ Aerospace Engineer, Aerodynamics Branch, NASA Dryden MS-2228, P.O. Box 273, Edwards, CA 93523, Member.
} 


\section{Introduction}

In the past several decades, the flight envelope has been expanding for military aircraft into high angles of attack and increased angular rates. Entry into increased angle-of-attack $(\alpha)$ regimes has consequently shifted the emphasis from steady-state, to quasisteady, ${ }^{1}$ to unsteady aerodynamics. With this shift, numerous complex problems associated with unsteady aerodynamics have come to the forefront. Problems such as flutter, buffet, abrupt wing stall, and wing rock adversely affect maneuverability and controllability of the aircraft. Although recent interest in solving unsteady aerodynamic problems has been spurred by military aircraft, civilian aircraft have had similar problems when subjected to adverse flight conditions such as crosswinds, gusts, and vortex encounters. ${ }^{2}$

The shift from quasisteady to unsteady aerodynamics lies partly in the inclusion of viscous effects. Viscous effects such as boundary layer transition from laminar to turbulent flow, flow separation and reattachment, and vortex dynamics play a large role in the performance, safety, and control of aerospace vehicles. For each of the unsteady aerodynamic problems mentioned earlier, there are associated viscous phenomena causing it: buffet can be caused by separated flow, inducing irregular oscillations to aircraft components; abrupt wing stall is caused by abrupt asymmetric flow separation on the wing, causing loss of lift on one wing; flutter can be caused by flow separation or shock impingement; and wing rock can be caused by asymmetric leading-edge vortices, inducing a limit-cycle oscillation in roll.

Traditionally, viscous effects have been very difficult to measure, yet arguably are the most untapped source of information for aerodynamics study. Large-scale flow structures emanate from the boundary layer. Observing the off-surface flow phenomena is useful in a qualitative manner, but probing the phenomena as it develops in the boundary layer provides the opportunity to understand fundamental flow physics as well as the ability to profoundly influence the resulting flow structures through small-scale modifications to the boundary layer.

Conventionally, instruments such as strain gages, accelerometers, pressure transducers, pressure sensitive paint, and even microphones are used to measure flow state and relevant aerodynamics. Each has distinct disadvantages. Strain gages and accelerometers indirectly derive the aerodynamic forcing function by measuring structural response. For load balance measurements, structural response of the system is only as accurate as the model used to map the measured strains to the imposed loads. Pressure-based instrumentation is typically challenging to set up and is subject to pneumatic latencies. Although pressure sensitive paint provides continuous spanwise pressure information, it suffers the same problems as other pressure-based instrumentation: significant setup time and strict temperature conditions. ${ }^{3}$ For unsteady wind tunnel tests, each of the above instrumentation types has major issues that limit its use. Temperature, pressure, and density are just a few of the environmental variables that change often in flight, so it would be beneficial to use a measurement technique that requires the minimal amount of calibration possible.

Hot-film anemometry can measure viscous effects like shear stress. This same method can also be used to characterize viscous phenomena such as transition, flow separation and reattachment, turbulence, and shock impingement. Hot-film sensors are also nonintrusive and can be installed on bodies without major structural changes. However, conventional anemometry has certain limitations. The large time constant of hot films (as opposed to hot wires) makes constant current anemometry unsuitable for use with hot films. ${ }^{4}$ Constant temperature anemometry can handle the large time constant but is complex to set up. An improvement upon conventional anemometry is the constant voltage anemometer (CVA). This instrument has a simple setup and high sensitivity with large bandwidth, ${ }^{5,6,7}$ therefore, it is an excellent choice for this application.

Another advantage of using hot-film anemometry for unsteady loads measurement is the ability to use a flow diagnostic technique called phase-reversal signatures (PRS), ${ }^{8-12}$ that does not depend on the absolute values of shear stress, thereby significantly reducing the need for calibration. Monitoring the PRS with the CVA enables quantitative in-flight measurement of the flow state in real-time.

This paper describes an approach that allows quantitative measurement and tracking of surface flow phenomena in order to obtain loads with minimal calibration requirements. This approach can even be used in the presence of changing environmental variables such as temperature and pressure, since the basic components of the technology

behind the approach have been demonstrated in flight. ${ }^{12,13}$ This paper presents a description of the wind tunnel setup (model, instrumentation, and data acquisition details), data analysis results, and interpretation of the unsteady aerodynamic data.

\section{Theory}

The objective of this paper is to make use of the relationship between surface flow phenomena, leading-edge stagnation point (LESP) and flow separation point (SEP), and aerodynamic loads. The important step then, is to 
develop that relationship empirically by correlating LESP and SEP with aerodynamic loads. The theory of obtaining a polynomial relationship as a correlating function of LESP and SEP to aerodynamic loads can be traced to a mathematical state-space representation by Goman and Khrabrov. ${ }^{14}$ Goman and Khrabrov describe the mathematical state-space representation for the unsteady aerodynamic problem as:

1. State Equation

$$
\tau_{1} \dot{x}+x=x_{0}\left(\alpha-\tau_{2} \dot{\alpha}\right)
$$

2. Output Equation

$$
C_{L}(\alpha, x)=\frac{\pi}{2}(1+\sqrt{x})^{2} \alpha
$$

where, $x_{0}$ is the nonlinear, steady-state dependency of SEP on $\alpha, x$ is the instantaneous location of the SEP, $\tau_{1}$ is the relaxation time constant which defines the transient aerodynamic effects, $\tau_{2}$ is the total time delay of the SEP and reattachment caused by quasisteady aerodynamic effects, such as the circulation and boundary-layer convection lags. The driving function is the steady-state location of the SEP, which is generally a nonlinear function of $\alpha$ and usually estimated from wind tunnel tests. From the output equation, the lift coefficient is shown as a function of $\alpha$ and instantaneous SEP.

In the above state-space model, for eq. 1, there are two unknown time constants that need to be determined using parameter estimation techniques with a large dataset consisting of experimental unsteady aerodynamic data at various unsteady flow conditions. For each condition, eq. 1 needs to be integrated to obtain a value for $x$. At the same condition, $\alpha$ and $x$ are input to eq. 2 which yields the lift coefficient.

This state-space method for determining unsteady loads has been validated by Goman and Khrabrov and Fan and Lutze $^{15}$ for various unsteady flow conditions involving flow separation and vortex breakdown using wind tunnel measurements on an airfoil, a flat delta wing, and an aircraft configuration. So, the state-space method allows the lift coefficient to be a function of only $\alpha$ and SEP.

Inspired by Goman and Khrabrov's work, if $\alpha$ and SEP are measured in real-time, then the lift coefficient can be computed directly from eq. 2 . The ability to directly measure $\alpha$ and SEP would, therefore, eliminate the need for extensive and expensive wind tunnel testing required to obtain the time constants. If LESP can be substituted for geometric $\alpha$, then hot-film sensors could be used to provide the necessary components for a polynomial approach. Hot-film sensors and the CVA can be used to provide real-time measurements of LESP and SEP. The LESP location is linearly proportional to geometric $\alpha$ in the prestall region. Near and after stall, LESP movement deviates from geometric $\alpha$. Instead of using the output equation, a first-order polynomial approximation of lift as a function of LESP and SEP is used. This approach can be intuitively justified by observing the relationship of the leading-edge and rear stagnation points with circulation. According to the Kutta-Joukowski condition, ${ }^{16}$ the circulation around an airfoil is determined by imposing the rear stagnation point to be located at the sharp trailing edge. When the rear stagnation moves upstream on the suction side because of flow separation, there is a significant loss in circulation which results in decreased lift and increased profile drag. So, intuitively, the LESP and SEP should be well correlated with circulation.

For this approach to work, the steady-state dependency of LESP and SEP on $\alpha$ needs to be measured to create a polynomial that correlates steady-state loads with the LESP and SEP flow features. Once the first-order polynomial is obtained, LESP and SEP data can be input into the polynomial equation to extrapolate the unsteady lift coefficient $\left(C_{L}\right)$. If the unsteady lift from the polynomial matches that from the pressure sensors then the method to measure loads from LESP and SEP is validated.

\section{Technical Approach}

Multiple hot-film sensors and the CVA were used to identify the LESP and SEP on a fixed and oscillatory NACA 0015 airfoil. A three-step approach was used in correlating LESP and SEP to unsteady loads on a NACA 0015 airfoil. The first step was to capture the flow features quantitatively through the airfoil stall region at steady-state and at different positive and negative pitch rates. The second step was to correlate the LESP and SEP with measured lift. The third and final step was to obtain a polynomial relationship between the LESP and SEP and 
the measured lift such that all observed unsteady cases are functions of the viscous phenomena. The United States Air Force Academy (USAFA) subsonic wind tunnel was utilized to set up the instrumentation, conduct several unsteady experiments, and collect data related to the viscous phenomena and lift.

\section{A. Model and Instrumentation Description}

The model tested was a symmetric 6-inch chord NACA 0015 airfoil. The airfoil was instrumented with 14 pressure ports and 104 hot-film sensors. Along one side of the airfoil, there were 14 pressure ports from approximately 2 percent to 90 percent chord and 70 hot-film sensors from approximately 1 percent to 97 percent chord. On the other side, the airfoil was instrumented with hot-film sensors 71 through 104 from approximately 1-percent to 40-percent chord. The leading edge is between sensors 70 and 71 . Figure 1 shows the instrumented airfoil within the test section.

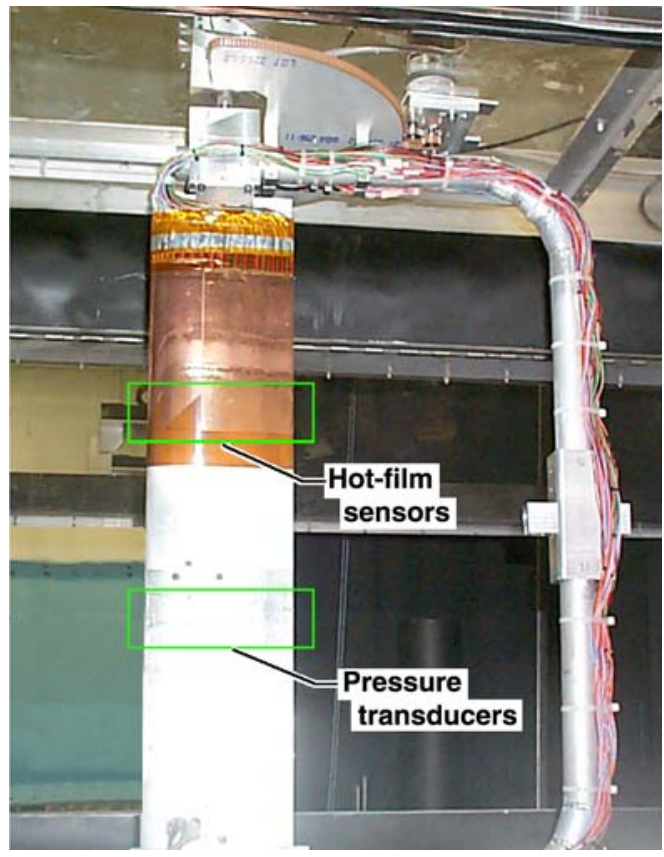

Figure 1. Instrumented airfoil in United States Air Force Academy subsonic wind tunnel.

An oscillating mechanism was constructed for the test. A proportional integral derivative (PID) controller was made to control the airfoil motion for accurate pitch rates. The angles were tracked using a quadrature encoder.

Once the instrumented NACA 0015 airfoil was integrated with the oscillating mechanism in the wind tunnel, the remaining associated instrumentation was connected. All 104 channels of hot-film sensors were each connected to a dedicated CVA. The CVA shear stress output was recorded onto analog tapes, along with the 14 pressure port data and the quadrature-encoded pitch angle. The hot-film, pressure, and angular data were recorded simultaneously onto analog tape and later digitized at a sampling frequency of $6 \mathrm{kHz}$ and $12 \mathrm{bit}$ analog-to-digital conversion.

\section{B. Experimental Approach}

The first step in the experimental approach was to capture the LESP and SEP quantitatively through the airfoil stall region at steady-state and at different positive and negative pitch rates.

Wind tunnel testing was performed at atmospheric conditions from speeds of $50 \mathrm{ft} / \mathrm{sec}$ to $100 \mathrm{ft} / \mathrm{sec}$. Data were obtained over the $\alpha$ range of minus $40 \operatorname{deg} \alpha$ to plus $40 \operatorname{deg} \alpha$. For the steady-state cases, the entire range of angles was stepped through at one-degree increments. Since there are only 14 pressure sensors on one side of the airfoil, the symmetry of the airfoil had to be utilized for determining the pressure profile for the upper and lower surfaces. For the positive angles of attack, the pressure sensors provided the pressure profile for the upper surface, and for the negative angles of attack the same pressure sensors provided the pressure profile for the lower surface.

For the unsteady cases, the oscillation mechanism was sinusoidally driven: 


$$
\alpha(t)=A(1-\cos (2 \pi f t))
$$

where geometric $\alpha$ is a function of time $t, A$ is the amplitude, and $f$ is the frequency. Three different frequencies were chosen and several amplitudes were used. The information in this paper is limited to two frequencies, $0.4 \mathrm{~Hz}$ and $0.8 \mathrm{~Hz}$, and one amplitude, $40 \mathrm{deg} \alpha$. One cycle consists of the oscillation mechanism starting at $0 \mathrm{deg}$, rising sinusoidally to $40 \mathrm{deg}$, and then dropping back to $0 \operatorname{deg} \alpha$. For the pressure sensors, the cycle must be mirrored for the other side as well, so that $\alpha$ goes from 0 deg to minus 40 deg back to 0 deg.

The LESP and SEP were identified primarily using PRS. However, as a redundant technique, the viscous phenomena were also identified by regions of minimum shear stress and the presence of a large root mean square (RMS) signal nearby. ${ }^{12}$

After the LESP and SEP had been identified and tracked, they were correlated to the measured lift. Lift was calculated by integrating the surface pressures on one side of the airfoil twice: once while at a positive $\alpha$ and then again at the same $\alpha$, except negative. The same technique was used to calculate lift from the unsteady pressure measurements. No latency corrections ${ }^{17}$ were made for the pressure measurements.

\section{Results}

First, a typical shear stress plot is shown to explain how the different flow features are represented in shear stress measurement. Then, graphs tracing the locations of LESP and SEP are shown, with combined plots of lift measured from integrating pressures along the airfoil. These graphs show how the LESP and SEP are correlated with measured lift. Using the LESP and SEP as input to a model, lift is calculated and compared with measured lift. Also, shear stress plots are shown for different pitch rates to exhibit dynamic stall.

\section{A. Labeled Shear Stress Plot}

Figure 2 illustrates an example pointing out the different flow features to be tracked and analyzed. The shear stress output in Fig. 2 is from the airfoil, as described earlier, instrumented with hot-film sensors on both the suction and pressure side, undergoing a pitchup then pitchdown motion from 0 deg to 40 deg and back to 0 deg $\alpha$. High shear stress regions are colored red and low shear stress regions are colored blue. Flow bifurcation points such as LESP and SEP exhibit low shear stress.

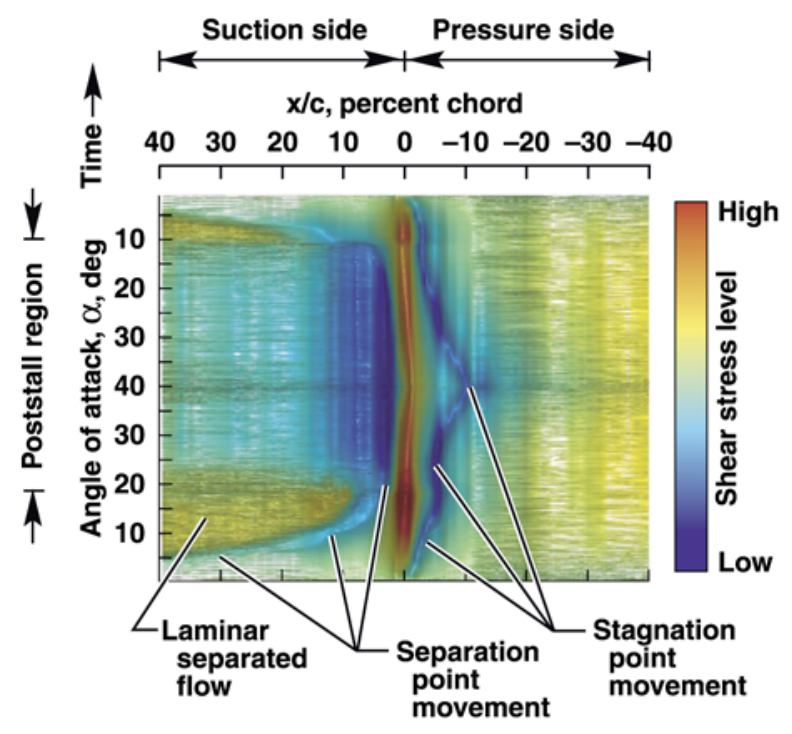

Figure 2. Shear stress distribution labeled with significant flow features $(100 \mathrm{ft} / \mathrm{sec}, 0.4 \mathrm{~Hz})$.

Focusing on the pressure side of the airfoil, observe the dark blue line moving initially from 0 percent chord at $0 \mathrm{deg}$ to approximately 10 percent chord at $40 \mathrm{deg}$ and then back to 0 percent chord at $0 \mathrm{deg}$. The differentiated line represents the instantaneous location and movement of the LESP. Now focusing on the suction side of the airfoil, 
observe another blue, very low shear stress line, moving initially from 40 percent chord at 45 deg to 0 percent chord at $40 \mathrm{deg}$ and then back to 40 percent chord at 3 to $4 \mathrm{deg}$. This line is the SEP movement. As labeled on the plot, the patches of yellow on the suction side represent laminar separated flow.

In Fig. 2 there are several trends to note concerning LESP and separated flow. For the positive pitch rate, the LESP moves away from the leading-edge linearly proportional to the increase in $\alpha$ to about 10 deg and then begins to recede around 15 to $20 \mathrm{deg}$. Stall occurs at approximately $18 \mathrm{deg}$ angle of attack. For the negative pitch rate, the LESP recedes at around $10 \mathrm{deg}$ angle of attack. When stall occurs, the LESP is close to receding or moving back towards the leading-edge. Compare when stall occurs for positive and negative pitch rates. Notice how stall occurs at higher angles of attack for the positive pitch rate than for the negative pitch rate.

With regard to the laminar separated flow, observe the relation between stall and the amount of laminar separated flow on the suction side. As $\alpha$ increases beyond stall, the SEP, darkest blue region on the suction side, moves toward the 0 percent chord resulting in leading-edge separation. The attached reversed flow from flow separation decreases rapidly beyond stall. Comparing the laminar separated flow for positive and negative pitch rate, at a positive pitch rate, the onset of leading-edge separation is delayed, and at a negative pitch rate, flow attachment is delayed.

\section{B. Stagnation and Separation Point Movement}

Although the LESP and SEP can be readily identified in a qualitative manner in the shear stress plots, for the purposes of this paper it is necessary to trace their motions precisely. This tracing is done using phase-reversal signatures (PRS). In Fig. 3(a), we can observe a 180-deg phase-shift between sensors 67 and 68 and the same for 89 and 91. The LESP is between 3.3- and 4.1-percent chord (sensors 67 and 68). The SEP is very close to sensor 90 which is at 18.2-percent chord, between sensors 89 and 91 (16.7- and 19.8-percent chord). Although it is not obvious from Fig. 3(a), there is a 180-deg phase-shift between sensors 90 and 91 . This can be clarified by calculating the cross-correlation coefficient for sensors 90 and 91 in Fig. 3(b). In Fig. 3(b), at zero lag, the correlation coefficient is negative, which is indicative of a 180-deg phase-shift between sensors 90 and 91. The figures trace LESP and SEP for $100 \mathrm{ft} / \mathrm{sec}$ and $0.4 \mathrm{~Hz}$.
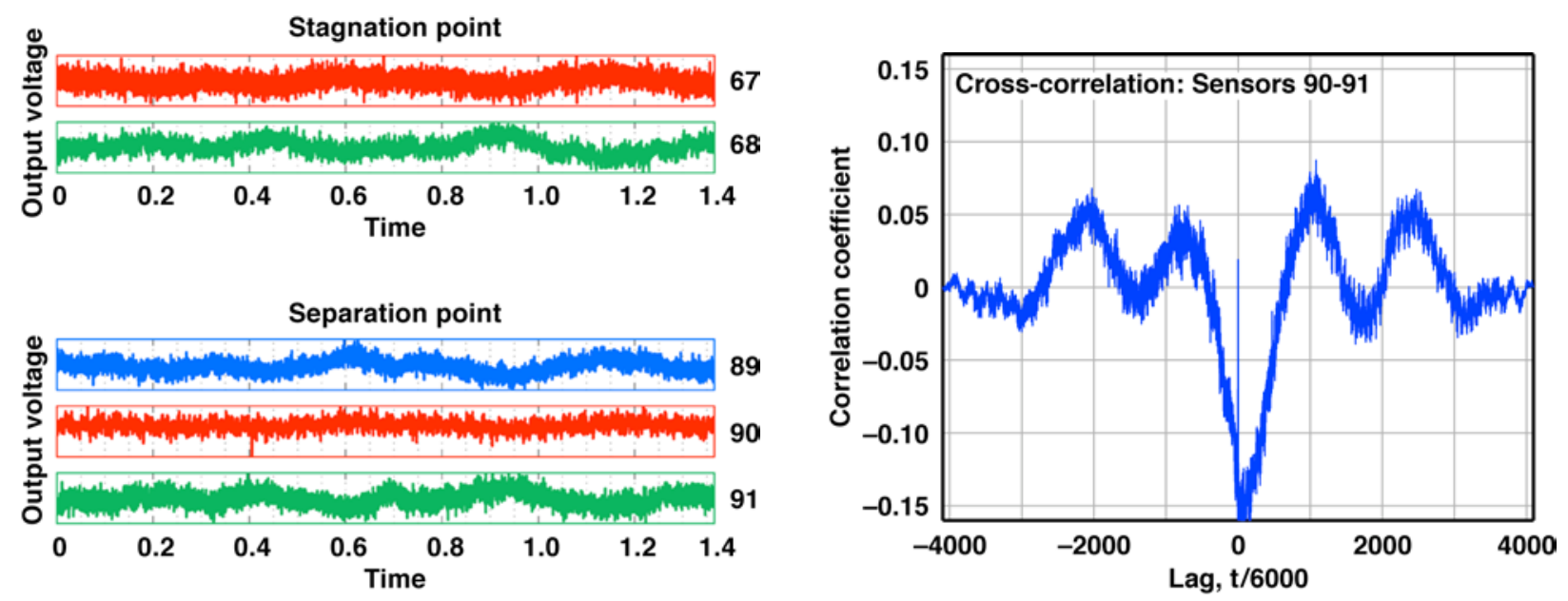

a) 180-deg phase-shift for stagnation and separation point.

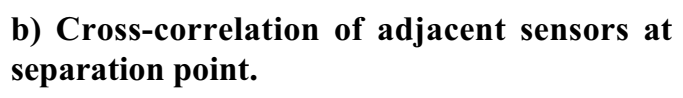

Figure 3. Phase-reversal signatures (PRS): 180-deg phase-shift and cross-correlation.

In Figs. 4(a) and 4(b), the LESP and SEP movements are plotted as chord locations as a function of the angle of attack. Lines are used for Fig. 4(a) to help show the trend. As compared to the steady-state LESP movement, the pitchup LESP movement overshoots and the pitchdown LESP movement undershoots. The steady-state LESP drops at close to $15-\operatorname{deg} \alpha$. The pitchup LESP drops much later at near 18-deg $\alpha$. The pitchdown LESP drops near 9-deg $\alpha$. Since the LESP is related to circulatory lift, it exhibits properties associated with dynamic stall. 


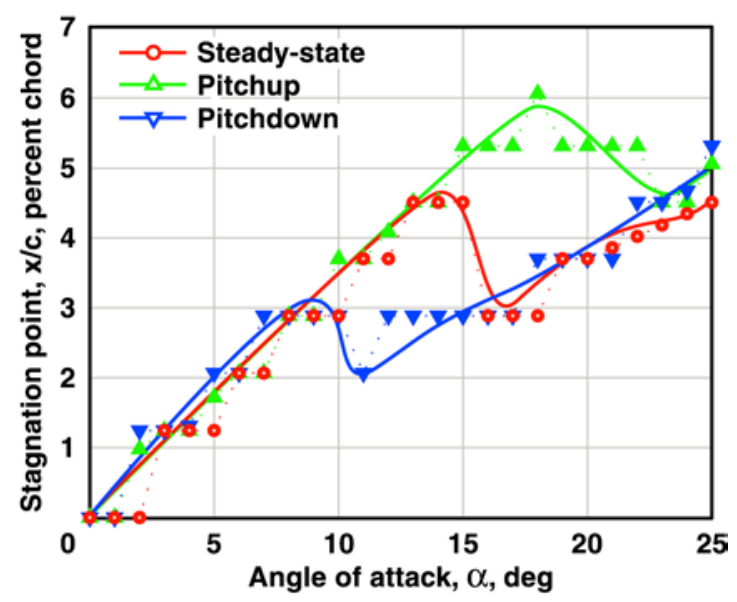

a) LESP movement.

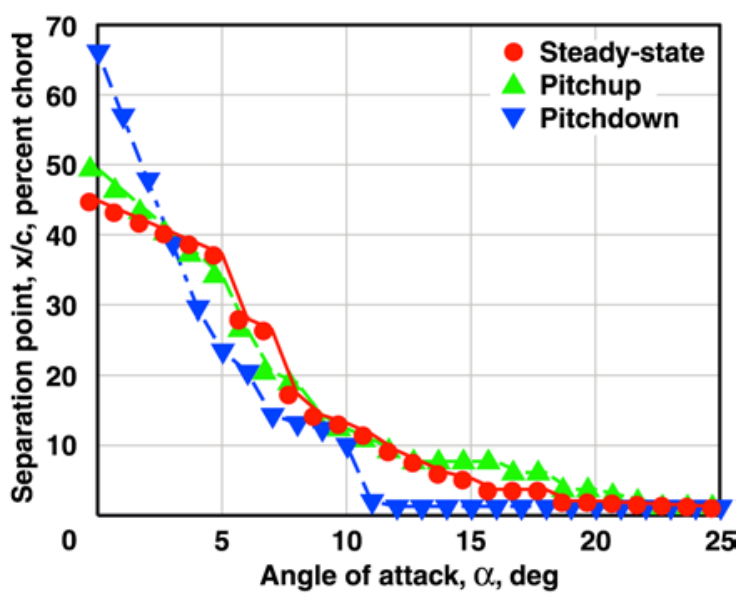

b) SEP movement.

Figure 4. LESP and SEP movement for steady-state, pitchup, and pitchdown (100 ft/sec, $0.4 \mathrm{~Hz})$.

The SEP movement shown in Fig. 4(b) shows how long flow takes to reattach for pitchdown. The pitchup SEP takes much longer to get to the leading edge, longer than even the steady-state SEP. So, leading-edge separation is delayed for the airfoil pitching up.

Figures 5(a) and 5(b) look very similar. Figure 5(a) is lift calculated from measured pressures for pitchup and pitchdown motions. Ignoring the pneumatic lag in the pressure output and the measured lift coefficient, one can see, by comparing Figs. 5(a) and 5(b), the clear correlation between the hysteresis in lift and the corresponding hysteresis in LESP movement.

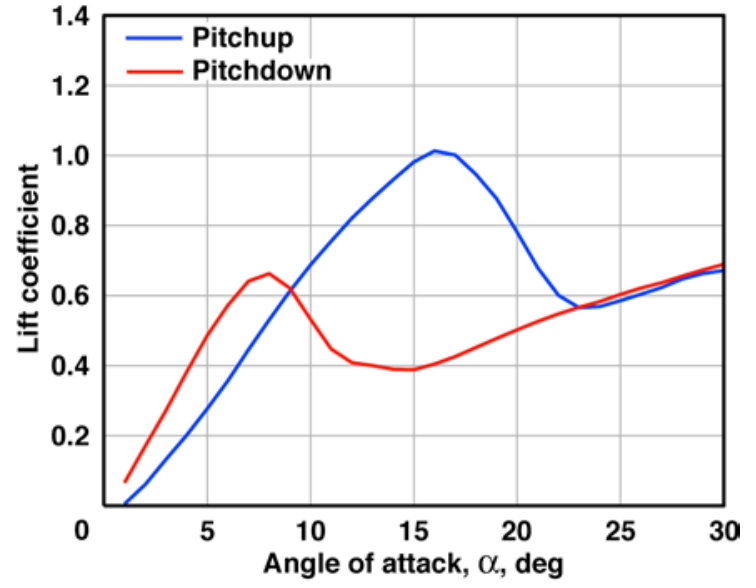

a) Lift measured from integrating pressures.

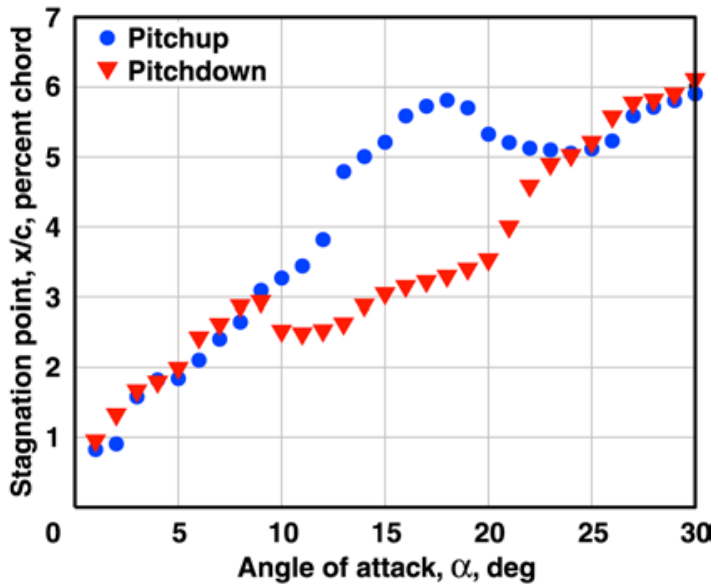

b) LESP movement.

Figure 5. Lift and LESP movement for steady-state, pitchup, and pitchdown (100 ft/sec, $0.4 \mathrm{~Hz})$.

Plotting the measured lift on top of the LESP movement clearly shows the correlation. In Fig. 6, the lift and LESP movement are plotted together. Figure 6(a) shows that the steady-state LESP movement follows lift linearly to the stall angle. Before stall, for an increase in lift coefficient of 0.2, the LESP moves approximately 1-percent chord away from the leading edge. The pitchup and pitchdown LESP movements in Figs. 6(b) and 6(c) have similar trends to the pressure-integrated lift. The next step is to use the LESP and SEP as inputs into a polynomial function that approximates the lift coefficient. 


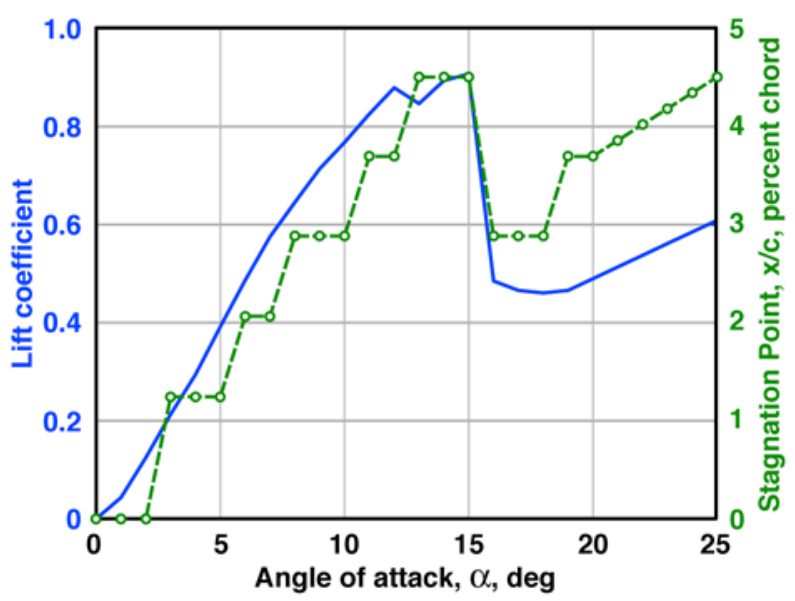

a) Steady-state.

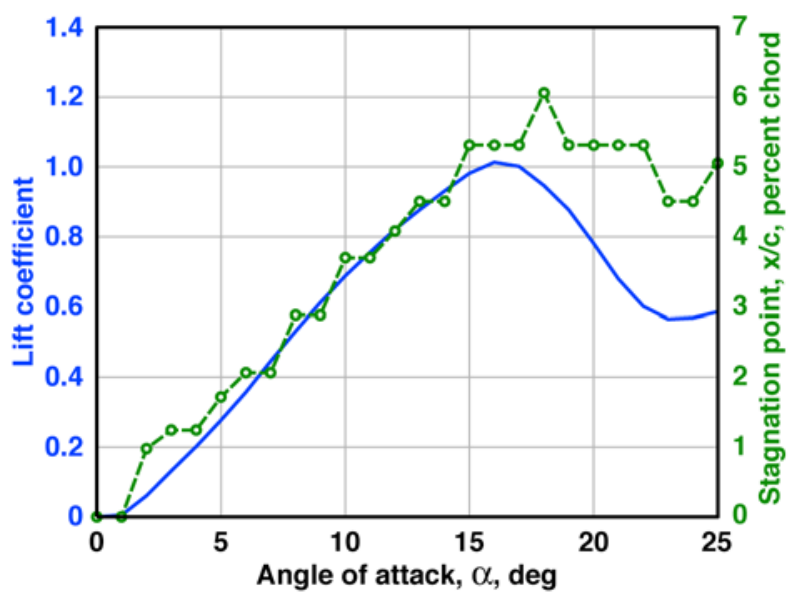

b) Positive pitch rate $(0.4 \mathrm{~Hz})$.

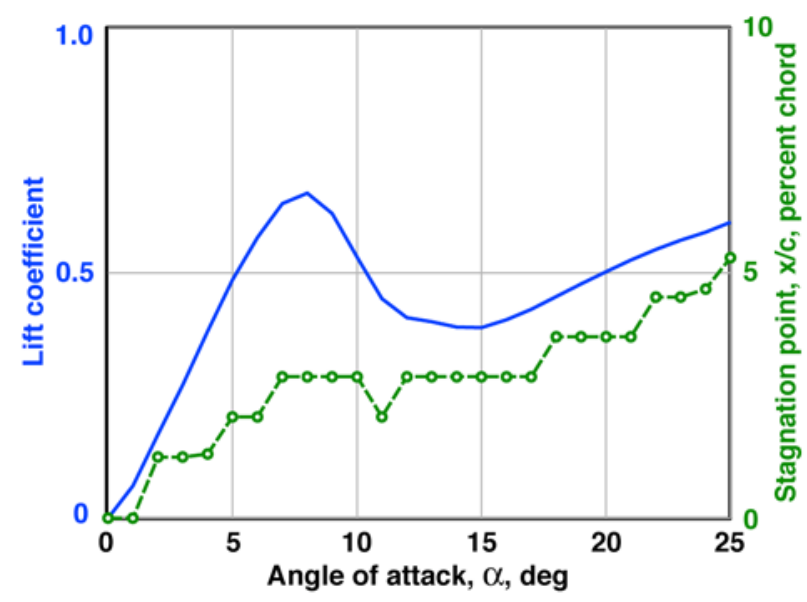

c) Negative pitch rate $(0.4 \mathrm{~Hz})$.

Figure 6. LESP movement and lift (100 ft/sec). 


\section{Lift Polynomial}

The following equation is a first-order polynomial of the lift coefficient as a function of LESP and SEP $x / c$ locations:

$$
C_{L}\left(x_{\text {lesp },} x_{\text {sep }}\right)=a_{0} x_{\text {lesp }}+a_{1} x_{\text {sep }}
$$

where, the $a$ coefficients need to be determined through a least-squares fit of steady-state input: LESP, SEP, and lift. A distinct prestall and poststall lift distribution was observed. Therefore, two polynomials were needed to describe the lift curve. Stall is empirically determined to be when SEP reaches 5-percent chord. Figure 7 shows both the resulting prestall and poststall polynomial equations for $100 \mathrm{ft} / \mathrm{sec}$. The prestall equation is:

$$
C_{L}\left(x_{\text {lesp }}, x_{\text {sep }}\right)=0.2051 x_{\text {lesp }}+0.0002 x_{\text {sep }}
$$

and the poststall equation is:

$$
C_{L}\left(x_{\text {lesp },} x_{\text {sep }}\right)=0.1113 x_{\text {lesp }}+0.0406 x_{\text {sep }}
$$

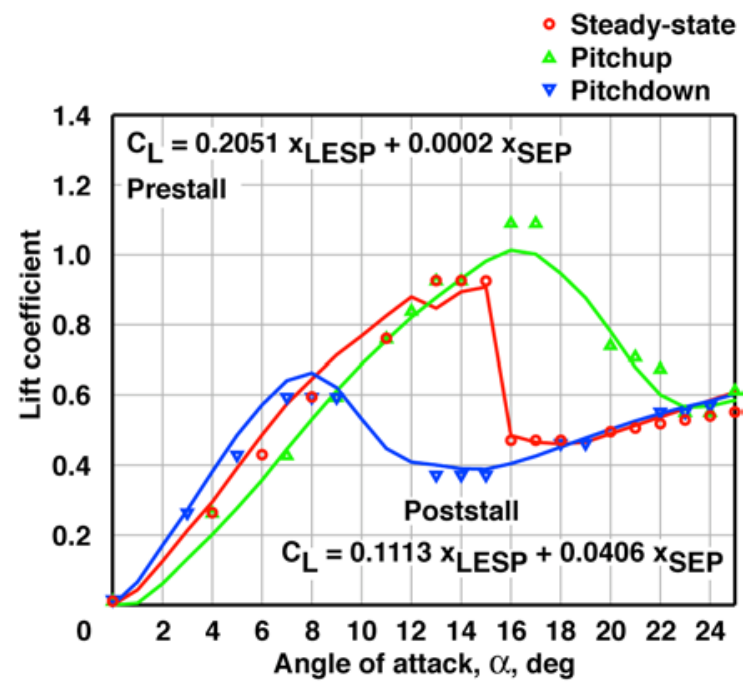

Figure 7. Comparison of pressure (solid lines) to polynomial fit (symbols) $(100 \mathrm{ft} / \mathrm{sec}, 0.4 \mathrm{~Hz})$.

The locations of LESP and SEP in the unsteady pitchup and pitchdown cases are then inserted into the above equations, as $x_{\text {lesp }}$ and $x_{\text {sep }}$ respectively, to predict lift, resulting in Fig. 7. The unsteady lift output in Fig. 7 clearly exhibits the same hysteresis as the lift curves. The predicted lift curves are not very far from the pressure-based lift coefficients. As a first cut, flow features such as LESP and SEP seem to represent the lift curves. Observe that for the prestall the polynomial equation, $a_{1}=0.0002$, is very small compared to $a_{0}=0.2051$. This observation shows that LESP has far more influence, in a least-squares sense, than SEP has on lift in the prestall angles of attack. Therefore, the LESP by itself can approximate the lift curves fairly well before stall occurs. The location at which LESP recedes towards the leading edge seems to be a precursor to stall. We shall use this observation in the next section when comparing increasing pitch rates.

\section{Increasing Pitch Rates}

The next issue is whether the flow features are consistent with published results as regards dynamic stall. At high positive pitch rates, increased lift is expected, and, at high negative pitch rates, decreased lift is expected. To verify whether or not the flow features are able to produce the expected trends for different pitch rates, two shear stress plots at two different frequencies are shown and analyzed. 
Figures 8(a) and 8(b) have labels on the left showing approximately where the LESP starts to recede for pitchup and pitchdown motions. For the positive pitch rates, the LESP recedes around $18 \operatorname{deg} \alpha$ for $0.4 \mathrm{~Hz}$ and $19 \operatorname{deg} \alpha$ for $0.8 \mathrm{~Hz}$. So, for positive pitch rates, increasing the pitch rate, increases the angle at which LESP recedes. For the negative pitch rates, the LESP recedes near $9 \operatorname{deg} \alpha$ for $0.4 \mathrm{~Hz}$ and $8 \mathrm{deg} \alpha$ for $0.8 \mathrm{~Hz}$. So, for negative pitch rates, increasing the pitch rate, decreases the angle at which LESP recedes. If we were to use the recession of the LESP as a precursor to stall, then at higher positive pitch rates, stall is delayed. Similarly, at high negative pitch rates, the airfoil stayed under stall conditions longer. For positive pitch rates, as the frequency increased, the flow was attached longer. For the negative pitch rates, as the frequency increased, flow reattachment was delayed further. Therefore, the LESP movement provides the trends of dynamic stall.

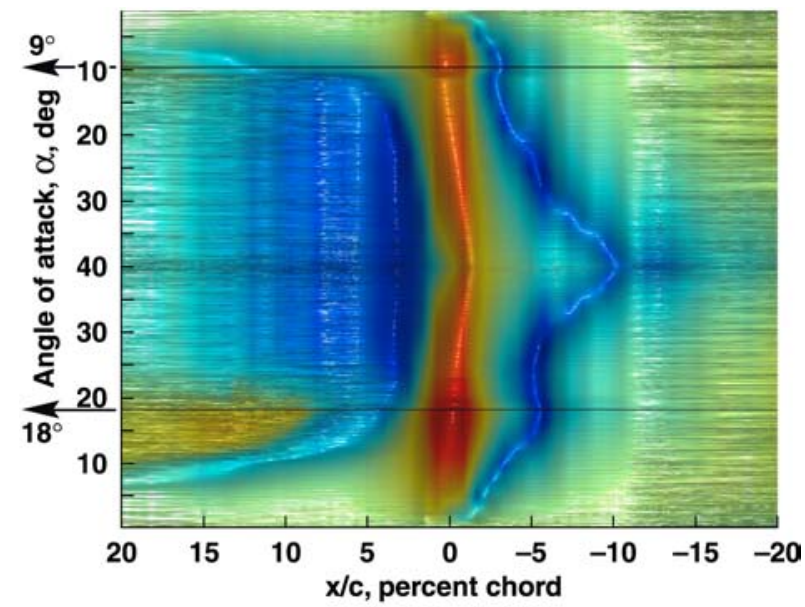

a) Pitch rate $(0.4 \mathrm{~Hz})$.

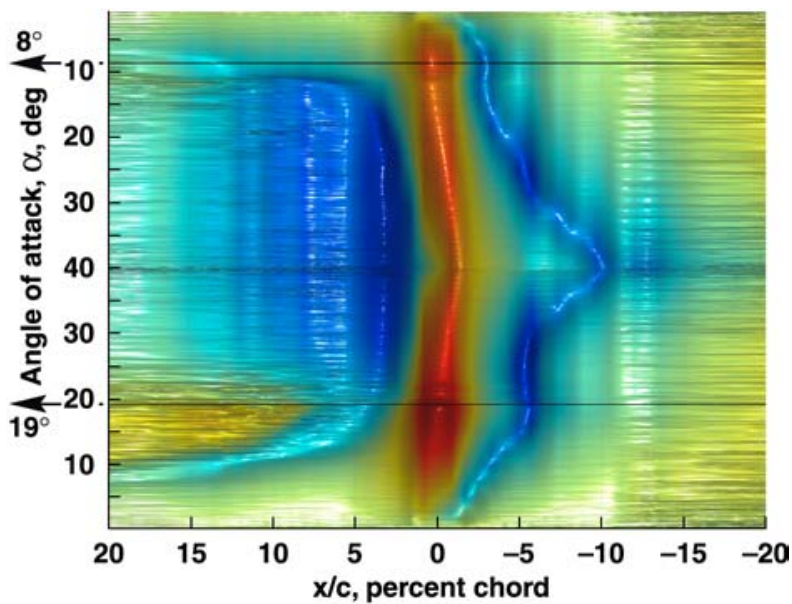

b) Pitch rate $(0.8 \mathrm{~Hz})$.

Figure 8. Shear stress profile: Increasing pitch rates $(100 \mathrm{ft} / \mathrm{sec}, 0.4 \mathrm{~Hz}$ and $0.8 \mathrm{~Hz})$.

\section{Concluding Remarks}

For a pitching NACA 0015 airfoil, leading-edge stagnation point (LESP) and flow separation point (SEP) movement was tracked using both shear stress plots and phase-reversal signatures (PRS). A steady-state polynomial relationship between lift and the locations of LESP and SEP was empirically developed. Using the steady-state polynomial relationship and the locations of LESP and SEP at airfoil pitchup and pitchdown, unsteady lift was extrapolated and compared to lift calculated from pressure sensors. This comparison showed the lift derived from the LESP and SEP tracked the lift calculated from pressure sensors. The LESP movement was further shown as similar to lift curves. The recession of LESP towards the leading edge was indicated to be a precursor to stall. The LESP recession at different pitch rates showed trends similar to that of dynamic stall. Such data indicate it is possible to obtain unsteady loads in real-time from LESP and SEP. These results have been obtained in incompressible flow. Further research needs to be conducted to consider compressible and Reynolds number effects.

Identifying and tracking flow features such as LESP and SEP in real-time provides a clear benefit in the unsteady aerodynamics field. Analytically, the tracked LESP and SEP can provide input to an empirical code that more accurately predicts unsteady effects. For wind tunnel and flight test studies, tracking LESP and SEP can be a way to capture any scale effects since the LESP and SEP can be accurately tracked in the wind tunnel and in-flight without any calibration. Any change in unsteady viscous behavior resulting from scale differences between the model and actual flight vehicle exhibits itself through LESP and SEP. Such changes can be recorded, analyzed, and incorporated back into empirical codes.

For flight experiments, tracking the LESP and SEP can allow for surface flow diagnostics that help understand and prevent abrupt wing stall, wing rock, onset of flutter, and buffet. Incorporating real-time flow diagnostics as an integrated smart sensor would enable the development of a closed-loop active flow control solution, allowing for new levels of performance and safety for both military and civilian aircraft. 


\section{References}

'Wang, Zhongjun, Lan, C.E., and Brandon, Jay M., "Unsteady Aerodynamic Effects of the Flight Characteristics of an F-16XL Configuration,” AIAA Paper 2000-3910, August 2000.

'Beyers, Martin E., and Ericsson, Lars E., "Flow Physics of Transport Aircraft in Crosswind Landing Maneuvers," AIAA Paper 2001-0693, January 2001.

3 McMillin, S.N., Hall, R.M., and Lamar, J.E., "Transonic Experimental Observations of Abrupt Wing Stall On an F/A-18E Model (Invited)," AIAA Paper 2003-591, January 2003.

"Comte-Bellot, Genevieve, "Hot-Wire Anemometry," Chapter 34 of Handbook of Fluid Dynamics, edited by Richard W. Johnson, Handbook Series for Mechanical Engineering, CRC Press, Boca Raton, FL, 1998.

'Comte-Bellot, Genevieve, and Sarma, Garimella R., "Constant Voltage Anemometer Practice in Supersonic Flows," AIAA Journal, Vol. 39, No. 2, February 2001, pp. 261-270.

${ }^{6}$ Comte-Bellot, Genevieve, Sarma, Garimella R., Faure, Thierry M., Dussauge, Jean-Paul, Dupont, Pierre, and Debieve, Jean-Francoise, "Performance Studies of the Constant Voltage Anemometer in a Mach 2.3 Boundary Layer," 18th International Congress on Instrumentation in Aerospace Simulation Facilities, Toulouse, France, June 14-17, 1999.

7 Sarma, Garimella R., "Analysis of a Constant Voltage Anemometer Circuit," IEEE Instrumentation and Technology Conference, May 1993, pp. 731-736.

${ }^{8}$ Stack, J.P., Mangalam, S.M., and Berry, S.A., "A Unique Measurement Technique to Study Laminar-Separation Bubble Characteristics on an Airfoil," AIAA Paper 87-1271. The $19^{\text {th }}$ AIAA Fluid Dynamics, Plasma Dynamics, and Lasers Conference, Honolulu, HI, June 8-10, 1987.

'Mangalam, S.M., Stack, J.P., and Sewall, W.G., "Simultaneous Detection of Separation and Transition in Surface Shear Layers." Fluid Dynamics of Three Dimensional Turbulent Shear Flows," AGARD CP-438, Paper no. 12. Presented at the Symposium on Fluid Dynamics Panel, Cesme, Turkey, October 3-6, 1988.

${ }^{10}$ Mangalam, S.M., and Kubendran, L.R., "Experimental Observations on the Relationship Between Stagnation Region Flow Oscillations and Eddy Shedding for Circular Cylinder," Instability and Transition: Materials of the Workshop held May 15-June 9, 1989 in Hampton, Virginia, Vol. 1, ICASE/NASA LaRC Series, M. Yousuff Hussaini and Robert G. Voight, eds., Springer-Verlag, 1990, pp. 372-381.

${ }^{11}$ Mangalam, S.M., Maddalon, D.V., Saric, W.S., and Agarwal, N.K., "Measurement of Crossflow Vortices, AttachmentLine Flow, and Transition Using Microthin Hot Films," AIAA Paper 90-1636, 1990.

${ }^{12}$ Mangalam, S.M., Wusk, M.S., and Kuppa, S., "In-Flight Detection of Stagnation, Transition and Separation Using Micro-Thin Surface Hot-Films," Society of Flight Test Engineers (SFTE) 22nd Annual Symposium Proceedings 1991.

${ }^{13}$ Moes, Timothy R., Sarma, Garimella R., and Mangalam, Siva M., Flight Demonstration of a Shock Location Sensor Using Constant Voltage Hot-Film Anemometry. NASA TM-4806, Aug. 1997.

${ }^{14}$ Goman, M., and Khrabrov, A., "State-Space Representation of Aerodynamic Characteristics of an Aircraft at High Angles of Attack," J. of Aircraft, Vol. 31, No. 5, Sept.-Oct. 1994, pp. 1109-1115.

${ }^{15}$ Fan, Yigang, and Lutze, Frederick H., "Identification of an Unsteady Aerodynamic Model at High Angles of Attack," AIAA Paper 96-3407, July 1996.

${ }^{16}$ Abbott, Ira H.A., and von Doenhoff, Albert E., Theory of Wing Sections. New York, NY: Dover Publications, 1959. 693 pp.

${ }^{17}$ Whitmore, Stephen A. and Moes, Timothy R., The Effects of Pressure Sensor Acoustics on Airdata Derived from a High-Angle-of-Attack Flush Airdata Sensing (HI-FADS) System, NASA TM-101736, Feb. 1991. 